\title{
Emprendimiento, dinámica empresarial y empleo: Una revisión desde la óptica del crecimiento económico
}

\section{Entrepreneurship, business dynamics and employment: A review from the Perspective of Economic Growth}

Rita De la Hoz-Villar

Fundación Universitaria Tecnológico Comfenalco

Cartagena - Colombia

ORCID iD: https://orcid.org/0000-0002-6460-225X

rdelahoz@tecnologicocomfenalco.edu.co

\section{Javier Prieto-Flórez*}

Universidad Tecnológica de Bolívar - Colombia

ORCID iD: https://orcid.org/0000-0002-6885-2790

jprieto@utb.edu.co

* Autor a quien debe ser dirigida la correspondencia
Fecha de recepción: 22/09/2019

Fecha de evaluación: 15/01/2020

Fecha de aceptación: 16/04/2020

Cómo citar: De la Hoz-Villar, R., \& Prieto-Flórez, J. (2020). Emprendimiento, dinámica empresarial y empleo: una revisión desde la óptica del crecimiento económico. Revista Científica Anfibios, 3(1), 11-18. https://doi.org/10.37979/afb.2020v3n1.57

\section{Resumen}

Con la complejidad del mundo globalizado actual resulta importante comprender las dinámicas de las organizaciones y como estas contribuyen a la dinámica económica. Es por esto que se pretende plantear una revisión de los artículos recientes que se han desarrollado en torno al Emprendimiento, la Dinámica empresarial y Empleo, desde la óptica del crecimiento económico. Para tales fines se comienza por dilucidar algunas teorías vinculadas con el emprendimiento y el crecimiento económico plateadas por los pensadores más importantes en cada campo, y a partir de allí se recopilan las investigaciones con mayor relevancia durante los últimos años que vinculen estas temáticas. Los hallazgos principales radican en una importancia vital del emprendimiento para el crecimiento económico, sin embargo, el aumento del empleo depende en gran medida de en qué sectores económicos se creen las empresas. Bajo este enfoque se plantean algunas conclusiones y se proponen discusiones que podrían dar origen a investigaciones futuras en la temática.

Palabras claves:

Emprendimiento; Crecimiento económico; Emprendedor; Empleo; Teorías del pensamiento económico

\begin{abstract}
With the complexity of today's globalized world, it is important to understand the dynamics of organizations and how they contribute to economic dynamics. This is why we intend to review the recent articles that have been developed around Entrepreneurship, Business Dynamics and Employment, from the perspective of economic growth. To this end, we begin by elucidating some theories linked to entrepreneurship and economic growth that have been put forward by the most important thinkers in each field, and from there we compile the most relevant research in recent years that links these issues. The main findings lie in the vital importance of entrepreneurship for economic growth, however, the increase in employment depends largely on which economic sectors companies are created in. Under this approach, some conclusions are drawn and discussions proposed that could give rise to future research on the subject.
\end{abstract}




\section{Keywords:}

Entrepreneurship; Economic growth; Entrepreneur; Employment; Economic thinking theories

\section{Introducción}

En la actualidad, la decisión de crear un negocio es un proceso complejo que implica incertidumbre y riesgo, por lo que está limitado por una serie de factores económicos, sociales, culturales e institucionales (León Mendoza, 2018), y son precisamente esos factores lo que permiten que esta generación de empresas aporte a la sociedad.

Un elemento fundamental para que las naciones sean dinámicas y gocen de bienestar social es el emprendimiento (Machado-Licona \& Franco-Blanco, 2016), para ello es necesaria la existencia de emprendedores con ideas de negocios encaminadas al crecimiento económico, estos empresarios den poseer competencias, habilidades, vocación y el potencial para hacer que su empresa crezca significativamente con el tiempo (Kantis et al., 2014).

La importancia del vínculo entre el emprendimiento, el empleo y el crecimiento económico radica en que el incremento del empleo indica el dinamismo de la economía y es un reflejo de la competitividad favorable en la división espacial del trabajo existente, mientras que el aumento del desempleo o las problemáticas que dificulten la inserción fuerza laboral refleja la situación opuesta (Méndez \& Prada, 2014). Por lo tanto, la dualidad de la iniciativa empresarial y el crecimiento económico se ha convertido en la agenda de los gobiernos de todo el mundo, lo que permite el desarrollo de nuevas intervenciones con un enfoque en el apoyo a las microempresas y las organizaciones de interés social o comunitario (Campuzano Vásquez et al., 2019).

Por lo anterior, el emprendimiento se ha convertido en una parte importante del crecimiento económico general, pasando de la dependencia grandes organizaciones a unas más reducidas, lo que está incrementando el número de organizaciones en términos generales en todos los países de Latinoamérica (Campuzano Vásquez et al., 2019). No obstante, no es posible desconocer las críticas ante la falta de evidencia del hecho que tiene mayor favorabilidad dirigir los esfuerzos e incentivos económicos gubernamentales a la relación emprendimiento y crecimiento centrado en la creación de nuevas empresas en vez de fortalecer las organizaciones más grandes (Audretsch \& Fritsch, 2002; Fritsch \& Mueller, 2004).

Tomando en consideración lo anterior, se propone estudiar la relación entre emprendimiento y crecimiento económico tomando en consideración el factor empleo, por lo tanto, se parte de un apartado teórico en donde se esboza a los aportes de los principales pensadores respecto al emprendimiento y la dinámica empresarial además de complementar este aspecto mencionando las teorías de crecimiento económico planteadas por las principales escuelas de pensamiento económico que vinculan al empleo como un factor fundamental para la dinámica económica. Desde esa óptica planteada se presentan las investigaciones recientes que han contribuido al entendimiento de la relación el emprendimiento, la dinámica empresarial y el empleo, de tal forma que se pueda obtener una visión holística de cómo estas variables confluyen para finalmente proponer conclusiones y discusiones.

\section{Apartado teórico}

\section{Emprendimiento y dinámica empresarial}

Desde que Cantillon en 1732 definió el emprendimiento como el deseo de los individuos de desplegar formas de intermediación que involucran un riesgo económico en la creación una empresas, diversos autores clásicos como John Stuart Mill, Joseph Schumpeter, y William Baumol han estudiado la relación entre la dinámica empresarial y su aportes a la economía y el empleo (Campuzano Vásquez et al., 2019; Minniti, 2012).

Para John Stuart Mill el aumento de capital, incremento de la población y ampliación de la producción son los tres elementos que conciben el progreso empresarial, enfatizando la relevancia que tiene el emprendimiento para el crecimiento económico (Primo Tapia \& Turizo Tapia, 2017)

Para Jean Baptiste Say existe una relación directa entre el empresario y la actividad industrial dado que en la época de este pensador se dio en con- 
texto de la industrialización francesa mecanizada de hilos de algodón (Primo Tapia \& Turizo Tapia, 2017), introduciendo la conceptualización de la administración y coordinación de recursos como uno de los tópicos principales en ese ámbito ya no solo aplicado a la industria si no en cualquier otro tipo de sector $\mathrm{u}$ organización.

Joseph Schumpeter centra su aporte en una óptica en la cual añade al sistema capitalista conceptos teóricos sobre el empresario, la innovación, el interés y el ciclo económico. Para Schumpeter, el emprendimiento y la dinámica empresarial constituyen los ejes fundamentales para el crecimiento económico, en la medida que el empresario es innovador y esto significa; la introducción de nuevos productos y servicios, nuevos métodos de producción, apertura de nuevos mercados; nuevas fuentes de materias primas y finalmente nuevas organizaciones en cualquier industria (Primo Tapia \& Turizo Tapia, 2017).

Por otra parte, Baumol (1990) considera que el emprendedor puede ser cualquier miembro de la economía que desarrolle actividades novedosas de cierta manera, utilizando elementos potenciales como ingenio, imaginación, liderazgo, audacia, determinación y perseverancia en busca de riqueza, poder y estatus, aunque no en ese orden especifico.

\section{El empleo como fuente de crecimiento económico}

Desde autores clásicos como Adam Smith, Jhon Srtuar Mill y David Ricardo entre otros hasta la actualidad, se han planteado una importante relación entre empleo y crecimiento económico que ha sido debatido en lo que hoy se conoce como escuelas de pensamiento económico (Piqué, 2018). Sin embargo, se referenciaran tres escuelas que se consideran las más notorias para explicar como el empleo incide en el crecimiento económico, siendo estas los neoclásicos, la escuela austriaca representada por Von Hayek y el Keynesianismo (Suárez Dávila, 2018).

Para economistas clásicos como Adam Smith y David Ricardo el elemento principal para el crecimiento económico de un país consiste en el aumento de la productividad laboral y esta a su vez depende del grado de especialización y división social del trabajo del territorio, concibiendo el análisis económico como un todo ori- entado por "la mano invisible del mercado" que equilibraba los precios y mercancías al interior de estos, sin embargo, con un contexto económico más desarrollado y una gran concentración de la producción resultado de los postulados de Smith, los neoclásicos con importantes pensadores como Menger, Jevons, Von Wieser, E. Bohm-Bawerk, Walras y Marshall dejaron los análisis de la economía como un todo y se centraron en los fundamentos microeconómicos de la formación de los precios, es así como las curvas de oferta y demanda se establecen a partir de las teorías de la productividad marginal y la utilidad marginal respectivamente, apoyándose en un sistema concurrente en donde todos los precios se igualan, estas fluctuaciones de precios flexibles conducirán automáticamente al uso completo de los factores de producción. haciendo que el libre funcionamiento del mercado condujera al pleno empleo.

Bajo este contexto aparece el pensamiento de Robert Solow con su modelo crecimiento endógeno, en el cual la producción depende la cantidad de mano de obra empleada y el capital utilizado, agregando el factor de la tecnología como una variable endógena dentro del modelo que permite mejoras en la productividad.

Posterior a estos pensadores, Von Hayek y la escuela austriaca consideran que el empleo depende del ciclo económico y que este a su vez está sujeto a las tasas de interés, esto se basa en que los empresarios, con una tasa inicialmente baja de interés, deciden invertir en proyectos arriesgados que en caso de una tasa más alta no hubieran emprendido, en corto plazo generan un aumento en empleo y producción, sin embargo, debido a los desequilibrio en el consumo y la inflación, posteriormente se genera una recesión que devuelve a la economía a su punto de ajuste. Estos dejan al gobierno de turno con herramientas monetarias, a través de la tasa de interés, para ajustar la economía.

En contraposición, la escuela Keynesiana considera que los mercados y sus equilibrios no generan automáticamente el pleno empleo, haciendo que se presenten en momento de crisis periodos extenso de desempleo, en ese orden de ideas, el gobierno debía aumentar la demanda agregada de bienes y servicios y de esa manera 
reactivar el empleo generando de esa manera el crecimiento económico.

En síntesis, se presentaron tres de las escuelas más importantes en lo relacionado con empleo y crecimiento económico con el ánimo de brindar un contexto en donde se puedan interpretar los resultados de investigaciones actuales vinculadas a emprendimiento, dinámica empresarial y empleo permitido proponer una revisión desde la óptica del crecimiento económico.

\section{Metodología}

Con el objetivo de brindar nuevas ópticas en la literatura organizacional, se inicia con una búsqueda sistemática de artículos que vinculen la temática del emprendimiento, dinámica empresarial y empleo desde una perspectiva del crecimiento económico tomando como referencia las publicaciones en bases de datos como Scopus y WOS, teniendo como criterios de selección; una fecha de publicación reciente; un alto impacto a través del índice de citación y revistas con factor de alto impacto y autores internacionales. De esta forma se estableció un marco teórico que por medio de los autores más relevantes en la temática pueda dar un contexto cognoscitivo en donde sea posible presentar los hallazgos más importantes de los artículos y de esa manera contribuir a la generación de nuevo conocimiento.
Posteriormente se propone una revisión analítica que permite considerar los hallazgos más interesantes en los campos teóricos y prácticos, por un parte, estableciendo conceptos teóricos que permitan esbozar un panorama actual de las corrientes de pensamiento que están siendo usadas en la literatura de gestión para soportar el crecimiento económico de los países o territorios por medio del empleo y el emprendimiento. Por otra parte, los aportes empíricos de los artículos en revisión permitirán conectar el estado actual de las empresas y el empleo con las corrientes de pensamiento de tal forma que se les de visibilidad a las últimas investigaciones realizadas en el campo investigativo.

Finalmente, a partir de este proceso se proponen conclusiones y discusiones en las cuales se plantean de manera sintetizadas los hallazgos más importantes registrados y se plantean temáticas todavía por investigar para que futuros

\section{Hallazgos}

En la siguiente tabla se pueden apreciar los aportes más significativos durante los últimos años vinculan la temática de Emprendimiento, Dinámica empresarial y Empleo desde la perspectiva del crecimiento económico.

Tabla 1. Emprendimiento, dinámica empresarial y empleo desde la perspectiva del crecimiento económico

\begin{tabular}{|c|c|c|c|}
\hline Autor & Año & Metodología & Resultados \\
\hline $\begin{array}{l}\text { - C a m p u z a n o } \\
\text { Vásquez, John } \\
\text { Alexander } \\
\text { Cedillo Chalaco, } \\
\text { Luis Fernando }\end{array}$ & 2019 & $\begin{array}{l}\text { Por medio de un estudio mixto de corte } \\
\text { descriptivo-exploratorio y con base en los } \\
\text { resultados del Doing Business 2018, el } \\
\text { Global Entrepreneurship Monitor } 2017 \text { y } \\
\text { las normas de emprendimiento en Ecua- } \\
\text { dor, se explora la relación entre empren- } \\
\text { dimiento y crecimiento económico en } \\
\text { Ecuador. }\end{array}$ & $\begin{array}{l}\text { Relación positiva entre emprendimiento y } \\
\text { crecimiento económico en Ecuador con la } \\
\text { generación de entorno empresarial favora- } \\
\text { ble para nuevos negocios en el país. }\end{array}$ \\
\hline $\begin{array}{l}\text { - Aguado Hernán- } \\
\text { dez, Juli Antoni }\end{array}$ & 2019 & $\begin{array}{l}\text { Desde un enfoque cuantitativo se analizan } \\
\text { datos del Ministerio de Empleo y Seguri- } \\
\text { dad Social y del Servicio Valenciano de } \\
\text { Empleo y Formación (España) con énfasis } \\
\text { en la medición del desarrollo económico } \\
\text { del territorio }\end{array}$ & $\begin{array}{l}\text { Gasto en políticas de empleo es incon- } \\
\text { secuente con el aumento del mercado } \\
\text { laboral, debido a desarticulación entre el } \\
\text { aparato estatal y la realidad económica del } \\
\text { territorio. }\end{array}$ \\
\hline $\begin{array}{l}\text { León Mendoza, } \\
\text { Juan }\end{array}$ & 2018 & $\begin{array}{l}\text { Enfoque cuantitativo y regresiones linea- } \\
\text { les múltiples en un modelo probit para los } \\
24 \text { departamentos de Perú, midiendo la } \\
\text { incidencia de las variables sociodemográ- } \\
\text { ficas en el nivel de creación de empresas } \\
\text { en el país. }\end{array}$ & $\begin{array}{l}\text { Las variables sociodemográficas determi- } \\
\text { nantes para la creación de negocios pro- } \\
\text { pios en Perú son la edad, situación laboral, } \\
\text { experiencia laboral, educación superior } \\
\text { alcanzada y tamaño de hogar. }\end{array}$ \\
\hline
\end{tabular}


- Zamora Boza, 2017 Clarisa Solange
Estudio analítico, de corte explotaría y descriptivo de la incidencia del emprendimiento en la dinámica económica de Ecuador y su política de entorno empresarial

- Gómez Sánchez, 2016 Andrés Mauricio

- Sarmiento Castillo, Juliana Isabel

- Fajardo Hoyos, Claudia Liceth para analizar la dinámica del mercado laboral en Popayán, Colombia
En términos generales la creación de empresas en Ecuador presenta un buen ritmo y esto impacta positivamente en la economía del país, sin embargo, no se cumplen las medidas para la generación de un entorno empresarial sostenible en el tiempo.

Desde una óptica económica e institucio- Limitaciones en generación de empleo nal y una revisión teórica se compilan da- estable debido a empresas basadas en el tos del DANE y la Cámara de Comercio sector comercial y de servicios

\section{Fuente: Elaboración propia de los autores.}

Tomando en cuenta que uno de los principales fenómenos que ha traído consigo la globalización es que ha permitido un proceso de descentralización de las actividades estatales dando mayor autonomía y responsabilidades a los entes territoriales, Aguado Hernández, (2019) explora el vínculo existente entre las principales variables del mercado de trabajo, la distribución y configuración social del empleo y las políticas activas de empleo en el País Valenciano durante los últimos 20 años haciendo énfasis especial en el presupuesto dedicado al emprendimiento del territorio.

Para tales fines, se expone brevemente que la crisis que atravesó España durante la recesión del 2008 afecto gravemente la calidad de empleo y el ratio de inserción laboral en el país, adicionalmente, la política de recortes que se implementó para aliviar el presupuesto de la nación en su intento por superar la crisis económica, afecto gravemente las políticas públicas de empleo, esto a su vez hizo que los entes territoriales españoles comenzaran a tener más autonomía y responsabilidad por sus políticas públicas, de tal manera que cada territorio adaptara el presupuesto y decisiones con el fin de responder a las necesidades específicas en materia laboral del departamento.

Este panorama tuvo como consecuencia que se presentaran desarticulaciones entre lo global y lo local, en la medida que presupuestalmente los territorios tenían cierta independencia, no obstante, se requerían de medidas más profundas que posibilitaran una verdadera articulación que posibilite un aumento del empleo y el emprendimiento dentro del territorio.
Por su parte, Campuzano Vásquez et al., (2019) propone la existencia de una relación entre la creación de empresas y el crecimiento económico a partir del impulso que proporcionan las políticas públicas y programas gubernamentales, esto basado en postulados de importantes pensadores económicos como John Stuart Mill, Jean Baptist Say, Joseph Schumpeter y William Baumol que conciben a las empresas como una herramienta fundamental para el crecimiento económico de los países.

En ese marco, se presentan los indicadores de emprendimiento y crecimiento económico en el Ecuador y asimismo las políticas públicas implementadas por el gobierno desde el año 2010 para incentivar un entorno empresarial amigable con las nuevas empresas y como este hecho influye en el crecimiento económico de la nación, dando así un sustento empírico de la importancia actual del emprendimiento como motor económico de los países, no obstante, es necesario conocer que variables intervienen en esos emprendedores.

Es por esto que León Mendoza (2018) analiza las variables sociodemográficas que inciden en el emprendimiento de negocios a nivel departamental en Perú, es por eso que se propone un modelo econométrico basado en la Encuesta Nacional de Hogares sobre Condiciones de Vida y Pobreza (ENAHO) del 2015 en ese país. Esta regresión se encuentra fundamentada en trabajos investigativos anteriores que determinan como variables significativas para la creación de empresas factores sociodemográficos como género (Doh \& Zolnik, 2011), edad debido a la madurez y aversión al riesgo que se genera en la adultez (Capelleras et al., 2013), 
condición de ser jefe de hogar dado que esto da capacidad de manejo y recursos en el hogar (Gluzmann et al., 2012), situación laboral (Neira et al., 2013), experiencia laboral (Xi et al., 2016), capital social (Neira et al., 2013), estado civil (Guerra \& Patuelli, 2016) entre otras.

Los resultados de este estudio apuntan a que tienen mayor probabilidad de crear una empresa las personas con mayor edad, posea empleo y mayor experiencia laboral y se relaciona negativamente con el tamaño del hogar. Esto hallazgos permitan dar una óptica individual del emprendimiento, sin embargo, también se requiere analizar la importancia del emprendimiento para la economía de los países.

Es así como Zamora (2018) destaca que a nivel mundial el interés de los gobiernos se ha centrado en el emprendimiento debido a su incidencia en incremento del empleo y en el crecimiento económico de los países, bajo ese marco, establece que, de acuerdo con su análisis del Global Entrepreneurship Monitor - Ecuador (2015), son los factores financieros, la capacidad emprendedora, las políticas de gobierno, educación, apertura de mercado, normas sociales y culturales y el clima económico, elementos que inciden positivamente en la creación de empresas y su respectivo impacto en el empleo. Por otra parte, la poca disponibilidad de capital semilla y excesiva regulación y legislación empresarial podrían anclar la creación de nuevas empresas en Ecuador.

De esta forma, se propone que los ámbitos en los cuales se pueden generar políticas de crecimiento económico que estimulen el emprendimiento y la disminución del desempleo radican en; un marco normativo que estimule desde el gobierno el espíritu empresarial; unas instituciones que vinculen como objetivos estratégicos el apoyo a las actividades emprendedoras; y programas de apoyo financiero para nuevas empresas.

Hasta el momento se han analizado diversas perspectivas que vinculan el emprendimiento con el crecimiento económico, sin embargo, los sectores en donde se desarrolla el emprendimiento también se encuentran muy relacionados con la habilidad de este para gen- erar empleo, es por esto que Gómez Sánchez et al., (2016) estudian la dinámica del mercado laboral de un territorio colombiano, teniendo como hallazgo principal que los sectores en donde se están generando las nuevas empresas como son el de servicios y comercio, ofrecen empleos muy inestables y por temporadas, esto indica que más allá del apartado gubernamental y las condiciones del entorno empresarial, el crecimiento económico al interior de los territorios depende en gran medida del renglón económico donde se desenvuelva estas nuevas empresas.

\section{Conclusiones y Discusiones}

Una de las herramientas más importantes con las que cuentas los entes gubernamentales para promover el crecimiento económico de sus territorios se encuentra en la generación de empleo por medio del emprendimiento (Zamora, 2018), esta conclusión se encuentra apoyada por muchos pensadores tanto en la literatura económica como la de gestión, aunque cada autor concibe de manera diferente como interactúan estos fenómenos. En ese sentido, las investigaciones abordadas dejaron en claro la relación entre estas tres variables en la medida que se incremente la creación de empresas también se podrá promover el empleo y a su vez el crecimiento económico (Alean Pico et al., 2017), sin embargo, esto depende en gran medida de los sectores donde se creen estas nuevas empresas, el entorno empresarial y el apoyo de políticas económicas efectivas que impulsen el espíritu empresarial.

Este marco genera algunas discusiones que pueden ser abordadas en futuros estudios investigativos, esto es, estudiar con mayor profundidad como la indecencia del emprendimiento en el crecimiento económico con el efecto mediador del empleo entre estas variables, proponiendo así un estudio de ecuaciones estructurales que soporte estadísticamente esa relación. Adicionalmente también se propone realizar estudios de prospectiva y desarrollo territorial que consideren la creación de empresas como una fuente para mejorar la situación de la población (Del Rio Cortina et al., 2019), asimismo, proponer escenarios en donde uno de los 
activos más importantes de las organizaciones sea la responsabilidad social empresarial, debido a que también esta considera algunas políticas vinculadas con la calidad de los empleos y mejores condiciones sociales para los trabajadores (Del Río-Cortina et al., 2017).
Por último, se plantea la necesidad de estudio que promuevan la competitividad y las estrategias diferenciadoras para nuevas empresas (Del Río Cortina et al., 2018), con el fin de generar valor al interior de las económicas y de esa manera promover el desarrollo social en los territorios.

\section{Referencias}

Aguado Hernández, J. A. (2019). La relación entre el mercado de trabajo y las políticas activas de empleo y desarrollo local en el País Valenciano (1997-2017): un estudio en curso. Lan Harremanak - Revista de Relaciones Laborales, 42, 149-177. https://doi.org/10.1387/lan-harremanak.21137

Alean Pico, A., Del Rio, J., Simancas Trujillo, R., \& Rodríguez Arias, C. (2017). ¿El emprendimiento como estrategia para el desarrollo humano y social? Saber, Ciencia y Libertad, 12(1), 107-123. https://doi.org/10.18041/2382-3240/saber.2017v12n1.1470

Audretsch, D. B., \& Fritsch, M. (2002). Growth regimes over time and space. Regional Studies, 36(2), 113-124. https://doi.org/10.1080/00343400220121909

Campuzano Vásquez, A. J., Chuquirima Espinoza, S. E., Romero Black, W. E., \& Cedillo Chalaco, L. F. (2019). Emprendimiento dinámico, crecimiento económico y política pública en el Ecuador Dynamic entrepreneurship, economic growth and public policy in Ecuador. INNOVA Research Journal, 4(3), 42-54. https://doi.org/10.33890/innova.v4.n3.1.2019.1076

Capelleras, J. L., Contín-Pilart, I., Martin-Sanchez, V., \& Larraza-Kintana, M. (2013). The influence of individual perceptions and the urban/rural environment on nascent entrepreneurship. Investigaciones Regionales, 26, 97-113.

Del Río-Cortina, J. L., Cardona -Arbeláez, D., \& Guacarí-Villalba, A. (2017). Responsabilidad social empresarial y construcción de la marca: una nueva mirada a las estrategias de gestión. Revista De Investigación, Desarrollo E Innovación, 8(1), 49. https://doi.org/10.19053/20278306.v8.n1.2017.7370

Del Rio Cortina, J., Arias, C. R., Espitia, N. L., \& Arbeláez, D. C. (2019). Prospective and territorial development in island areas: Empirical evidence from Bocachica-Colombia. Problems and Perspectives in Management, 17(4), 176-189. http://dx.doi.org/10.21511/ppm.17(4).2019.15

Del Río Cortina, J., Cardona Arbelaez, D., \& Pérez Olascuaga, S. J. (2018). Estrategias diferenciadoras y generadoras de valor en economías emergentes. Caso. Saber, Ciencia y Libertad, 13(1), 133-144. https://doi.org/10.18041/2382-3240/saber.2018v13n1.2083

Doh, S., \& Zolnik, E. J. (2011). Social capital and entrepreneurship: An exploratory analysis. African Journal of Business Management, 5(12), 4961-4975. https://doi.org/10.5897/AJBM11.095

Fritsch, M., \& Mueller, P. (2004). Effects of new business formation on regional development over time. Regional Studies, 38(8), 961-975. https://doi.org/10.1080/0034340042000280965

Gluzmann, P., Jaume, D., \& Gasparini, L. (2012). Decisiones laborales en América Latina: el caso de los emprendedores. Un estudio sobre la base de encuestas de hogares. CAF: Documento de Trabajo No. 2012/06, May 2014. https://scioteca.caf.com/handle/123456789/235

Gómez Sánchez, A. M., Sarmiento Castillo, J. I., \& Fajardo Hoyos, C. L. (2016). Análisis de la dinámica del mercado laboral en Popayán. Económicas CUC, 37(1), 137-178. 
Guerra, G., \& Patuelli, R. (2016). The Role of Job Satisfaction in Transitions Into Self-Employment. Entrepreneurship: Theory and Practice, 40(3), 543-571. https://doi.org/10.1111/etap.12133

Kantis, H., Gonzalo, M., \& Álvarez, P. (2014). Aprendizaje y del Ecosistema en la Creación de Nuevas Empresas Dinámicas. Pymes, Innovación y Desarrollo, 1(3), 33-60. https://dialnet.unirioja.es/servlet/articulo?codigo $=6353893$

León Mendoza, J. (2018). Emprendimiento de negocios propios en el Perú: el rol de los factores sociodemográficos personales a nivel de departamentos. Estudios Gerenciales, 34, 19-33. https:// doi.org/10.18046/j.estger.2018.146.2810

Licona, J. M., \& Blanco, L. F. (2016). Emprendimiento e innovación: estado del arte. Estrategia, 2(1), 73-86

Méndez, R., \& Prada, J. (2014). Crisis, desempleo y vulnerabilidad en Madrid. Scripta Nova: Revista Electrónica de Geografía y Ciencias Sociales, 18(474), 463-499.

Minniti, M. (2012). El emprendimiento y el crecimiento económico de las naciones. Economía Industrial, 1(383), 23-30. https://doi.org/10.15174/au.2014.701

Neira, I., Portela, M., Cancelo, M., \& Calvo, N. (2013). Social and human capital as determining factors of entrepreneurship in the Spanish Regions. Investigaciones Regionales, 26(26), 115-139.

Piqué, P. (2018). La enseñanza del proyecto filosófico de Adam Smith en la historiografía del pensamiento económico. Práxis Filosófica, 46, 11-41. https://doi.org/10.25100/pfilosofica.v0i46.6150

Primo Tapia, W. de J., \& Turizo Tapia, H. F. (2017). Emprendedor y empresario, una construcción desde la dinámica del pensamiento económico. Management Review, 2(1), 32. https:// doi.org/10.18583/umr.v2i1.44

Suárez Dávila, F. (2018). Un gran debate económico a través de nuestra historia, los liberales frente a los keynesianos desarrollistas. Economía UNAM, 15(45), 3-28.

Xi, G., Block, J., Lasch, F., Thurik, R., \& Lasch, F. (2016). Work Experience from Paid Employment and the Path to Entrepreneurship : Business Takeover versus New Venture Start-Up. Discussion Paper No. 9949, 9949.

Zamora, C. (2018). La importancia del emprendimiento en la economía: El caso de Ecuador. Espacios, 39, 20. 